\title{
Führungsaufsicht mit satellitengestützter Überwachung
}

\author{
Rita Haverkamp, Andreas Schwedler, Gunda Wößner
}

Seit dem 1. Januar 2011 ist die elektronische Aufenthaltsüberwachung von als gefährlich eingeschätzten Entlassenen aus dem Strafvollzug bundesweit zulässig. Die Kontrolle von Aufenthaltsverboten im Rahmen der Führungsaufsicht ${ }^{1}$ erfolgt mit Hilfe satellitengestützter Technik und soll die Sicherheit potenzieller Opfer (z. B. Kinder) erhöhen, indem Ausschlusszonen die Betroffenen in ihrer Mobilität außerhalb ihres Wohnraums einschränken. Die Einführung dieser Weisung geht auf die Reform der Sicherungsverwahrung im Anschluss an das Urteil des EGMR vom 17. Dezember $2009^{2}$ zurück, nach dem die Vollstreckung von Altfällen in Deutschland konventionswidrig ist und das daher Entlassungen aus dem Maßregelvollzug nach sich zog. Im Unterschied zum elektronisch überwachten Hausarrest, der die technische Kontrolle eines Ausgangsverbots betrifft, ermöglicht die elektronische Aufenthaltskontrolle eine Rundumüberwachung außerhalb des Wohnraums. ${ }^{3}$ Die neue Weisung weckt nicht nur Bedenken hinsichtlich ihrer Verfassungsmäßigkeit, denen hier nicht nachgegangen wird, sondern wirft auch Fragen zur Eignung der Zielgruppe, zu den (nicht) beabsichtigten Wirkungen, zur technischen Funktionstüchtigkeit in der Praxis und zum Anwendungspotenzial hierzulande auf.

\section{Überwachung mit GPS-Technologie}

Die elektronische Aufenthaltskontrolle erfordert den Einsatz satellitengestützter Technik, um die Einhaltung von Exklusionszonen computergestützt zu überprüfen. Zusätzlich wird Mobilfunktechnik zur Standortbestimmung in nicht satellitenüberwachten Bereichen (z. B. U-Bahn) und zur Datenübermittlung verwendet. Der Überwachte trägt ein Fußband mit einem GPSEmpfänger und einem Modul für Mobilfunk oberhalb des Knöchels. Das Fußband ist wasserfest, manipulationssicher und für Allergiker geeignet. ${ }^{4}$ In kurzen Abständen gibt dieses Fußband Signale an eine Überwachungszentrale $a b$, die dort automatisch mit den Standortdaten der angeordneten und im System vermerkten Gebots- und Verbotszonen abgeglichen werden. Hierzulande verwaltet die Hessische Zentrale für Datenverarbeitung (HZD) die pseudonymisierten Geodaten. Für die Durchführung der elek- tronischen Aufenthaltsüberwachung ist die Gemeinsame elektronische Überwachungsstelle der Länder (GÜL) zuständig, die die Ereignismeldungen (z. B. Weisungsverstoß oder Funktionsstörung) entgegennimmt und bewertet. ${ }^{5}$ Abhängig vom Ergebnis der Bewertung kontaktiert die GÜL den Überwachten, informiert die zuständigen Stellen der Länder (Polizei, Bewährungshilfe, Führungsaufsichtsstelle) zur Einleitung von Maßnahmen oder veranlasst eine Überprüfung der Funktionstüchtigkeit bzw. einen Austausch der GPS-Fußfessel. Wird ein einteiliges GPS-Gerät verwendet, so ist die Installation einer "Wohnungseinheit" in der Wohnung erforderlich ( $\$ 463$ a Abs. 4 S. 1 Hs. 2 StPO). Die Wohnungseinheit hindert die Übertragung von Positionsdaten innerhalb des Wohnraums, so dass eine reine Aufenthaltsfeststellung zu Hause erfolgt. ${ }^{6}$ Bei zweiteiligen GPS-Geräten, bestehend aus einer Fußfessel und einem Transmitter zur Übertragung der Koordinaten an die Überwachungszentrale, unterbleibt eine Weitergabe innerhalb des Wohnbereichs, weil sich der Transmitter auf einer Station in der Wohnung befindet.

\section{Weisung im Rahmen der Führungsaufsicht}

Die elektronische Überwachung des Aufenthaltsortes wurde am 1. Januar 2011 als neue Weisung im abschließenden Katalog der Führungsaufsicht nach $\mathbb{S} 68$ b Abs. 1 S. 1 Nr. 12 StGB eingeführt. ${ }^{7}$ Die Weisungen in $\ 68$ b StGB dienen der Unterstützung der Resozialisierung, dem Schutz der Gesellschaft und der Kontrolle des Verurteilten. ${ }^{8}$ Es handelt sich um strafbewehrte Weisungen, bei denen die Nichteinhaltung zur Strafbarkeit nach $\ 145$ a StGB führt. Die neue Weisung kann ohne Einwilligung des Überwachten für die Dauer der Führungsaufsicht ${ }^{9}$ oder für eine kürzere Zeit angeordnet werden. Der Betroffene ist verpflichtet, die technische Ausrüstung ${ }^{10}$ jederzeit in betriebsbereitem Zustand bei sich zu führen und deren Funktionstüchtigkeit zu gewährleisten. Die Funktionstüchtigkeit bezieht sich auf den Verzicht auf Manipulationen am Gerät und die Aufrechterhaltung der Energiezufuhr, wozu das tägliche zweistündige Aufladen der Batterie gehört. ${ }^{11}$
Die Legislative erhofft sich von dem Instrument hauptsächlich eine Erhöhung der Kontrolle und der Effizienz der Überwachung. Zusätzlich soll die elektronische Aufsicht „eine [...] Unterstützung zur Eigenkontrolle darstellen bzw. den Anreiz für den Betroffenen erhöhen, psychologisch vermittelte, nachhaltig wirkende Verhaltenskontrollen zu erlernen und zu verfestigen " ${ }^{12}$. Da vermutlich die Entdeckungs- und Sanktionswahrscheinlichkeit bei erneuter Begehung einer Straftat i. S. d. $\$ 66$ Abs. 3 S. 1 StGB durch die Erfassung der Positionsdaten steigt $^{13}$, soll der Überwachte das Bewusstsein um die eigene Gefährdung verinnerlichen und derart Selbstkontrolle einüben. Hier geht es um die Abschreckung vor der Begehung bestimmter Rückfalltaten im Sinne einer positiven und negativen Spezialprävention. $^{14}$

Die Zielgruppe erfasst sowohl Entlassene der Sicherungsverwahrung ${ }^{15}$ als auch Vollverbüßer mit einer mindestens dreijährigen (Gesamt-)Freiheitsstrafe, einer Katalogtat i. S. d. $\$ 66$ Abs. 3 S. 1 StGB und einer negativen einschlägigen Gefährlichkeitsprognose ( $\mathbb{S} 68$ b Abs. 1 S. 3 StGB). Bedeutung hat die elektronische Aufenthaltskontrolle insbesondere bei der Weisung, den Wohn- und Aufenthaltsort oder einen bestimmten Bereich nicht ohne Erlaubnis der Führungsaufsicht zu verlassen $(\$ 68$ b Abs. 1 S. 1 Nr. 1 $\mathrm{StGB}$ ) sowie bei der Weisung, sich nicht an bestimmten Orten aufzuhalten, die Gelegenheit oder Anreiz zu bestimmten Straftaten bieten können $(\mathbb{S} 68$ b Abs. 1 S. 1 Nr. 2 StGB). Nach der gesetzgeberischen Intention ist die elektronische Überwachung auch bei Kontakt- und Verkehrsverboten $(\$ 68 \mathrm{~b}$ Abs. 1 S. 1 Nr. 3 StGB) statthaft. ${ }^{16}$ In solchen Fällen wird eine mittelbare Wirkung vermutet, da lediglich der Aufenthaltsort und eben nicht die Aktivitäten des Betroffenen überwacht werden können. Schließlich kann die elektronische Aufenthaltsüberwachung als isolierte Weisung angeordnet werden, wenngleich in der Praxis regelmäBig die elektronische Aufenthaltskontrolle mit Mobilitätsweisungen verbunden werden dürfte.

Die verhaltenssteuernde Überwachung soll der vermuteten Rückfallgefahr entgegensteuern. Die neue Weisung impliziert somit die Compliance von einem als unverbesser- 
lich geltenden Überwachten, dessen Einwilligung aber für deren Anordnung überflüssig ist. Die fehlende Einwilligung weckt Bedenken hinsichtlich der Einhaltung der Weisung seitens des Überwachten. Zudem kann auf die Einholung eines Sachverständigengutachtens über die anhaltende Gefährlichkeit des Betroffenen grundsätzlich verzichtet werden, nur in Ausnahmefällen ist eine externe, sachverständige Person hinzuziehen. Als hinreichende Beweisgrundlage für eine Gefährlichkeitsprognose werden Stellungnahmen des psychologischen Dienstes der Justizvollzugsanstalt erachtet, da es an der Vergleichbarkeit zwischen elektronischer Überwachung und freiheitsentziehender Maßregel in Bezug auf die Eingriffsqualität fehle. ${ }^{17}$ Vor dem Hintergrund der zulässigen lebenslangen Dauer und der nicht notwendigen Einwilligung kann das Absehen von einem externen Sachverständigengutachten durchaus mit Skepsis betrachtet werden. Die Zweifel werden durch die herabgesetzten Anforderungen („erscheinen“) an die Erforderlichkeit der elektronischen Aufenthaltsüberwachung in $\$ 68$ b Abs. 1 S. 3 Nr. 4 StGB verstärkt, um den Verurteilten wegen der möglichen Datenverwendung nach $\$ 463$ a Abs. 4 S. 2 StPO von der Begehung weiterer Straftaten der in $\$ 66$ Abs. 3 S. 1 StGB genannten Art abzuhalten. Die Wortwahl „erscheinen“ signalisiert, keine überspannten Ansprüche an die Überzeugungsbildung für die Erforderlichkeit der Weisung zu stellen. Der Verzicht auf ein Sachverständigengutachten und das wachsweiche Kriterium der Erforderlichkeit könnten in der Praxis zur übereilten Anordnung der elektronischen Aufenthaltskontrolle führen und damit die intendierte restriktive Anwendung - Beschränkung auf schwere Katalogtaten i. S. d. $\$ 66$ Abs. 3 S. 1 StGB konterkarieren.

\section{Die Gefahr der Ausdehnung der Maßnahme}

Darüber hinaus lassen frühere Erfahrungen mit kontrollverstärkenden kriminalpolitischen Maßnahmen eher auf eine Ausdehnung des Anwendungsbereichs auch dieser Gesetzesnovelle schließen, obgleich die neu eingeführte Weisung „hinsichtlich des erfassten Personenkreises an enge Voraussetzungen geknüpft" ${ }^{18}$ wird.

Schon die Gesetzesänderung aus dem Jahre 1998, mit der die Legislative die Zehnjahresfrist im Rahmen der Sicherungsverwahrung abschaffte ${ }^{19}$, wurde unter der Annahme eingeführt, sie würde nur in wenigen
Ausnahmefällen, nämlich bei extrem gefährlichen Straftätern, Anwendung finden. Im Jahre 2008 befand sich allerdings jeder sechste Untergebrachte länger als zehn Jahre in der Sicherungsverwahrung. Auch von der Einführung der nachträglichen Sicherungsverwahrung waren nicht nur wenige Ausnahmefälle, wie es postuliert wurde, betroffen. Vielmehr wurden alleine zwischen 2006 und 2008 tatsächlich 572 Verfahren zur nachträglichen Sicherungsverwahrung eingeleitet. ${ }^{20}$ Diese Erfahrungen lassen also eher eine breitere Anwendung der neuen Weisung erwarten. Denn nach wie vor stellt die Gesetzgebung den „Schutz der Bevölkerung vor rückfallgefährdeten Straftätern“ ${ }^{21}$ und das „Sicherheitsinteresse der Allgemeinheit“ ${ }^{\prime 22}$ an erste Stelle. Fast scheint es, als ob durch dieses „Angstmanagement" ${ }^{\text {"23 }}$ der Resozialisierungsaspekt zur Nebensache gerate. $^{24}$

\section{Das Problem der \\ Gefährlichkeitsprognose}

Daher verwundert es nicht, dass keine überspannten Ansprüche an die Überzeugungsbildung für die Erforderlichkeit der Weisung gestellt werden müssen. Der Beifang der Prognoseunsicherheit wird gewissermaßen als „Sonderopfer“ in Kauf genommen, wenn die fälschlicherweise als gefährlich Eingeschätzten, aber tatsächlich nicht mehr Rückfallgefährdeten (false positives) auch im Netz der Maßnahme landen. Dabei sind mehrere Aspekte kritisch zu diskutieren. So ist die hypothesenbestätigende Wahrnehmungsselektion („,confirmation bias“) bei anstaltsinternen Stellungnahmen zu berücksichtigen. Denn ohne den Einspruch aus Straßburg würden die sogenannten „Parallelfälle“ ja weiterhin in der Sicherungsverwahrung verbleiben. Auch für externe Sachverständige scheint die Feststellung fortbestehender Gefährlichkeit bei Sexualstraftätern, „die oft während ihrer Unterbringung kaum prognoserelevante Auffälligkeiten nach außen hin deutlich werden lassen “25, fast unmöglich. Die Hypothese, ein bestimmter Gefangener sei nach wie vor gefährlich, kann somit quasi überhaupt nicht entkräftet werden, die Entlassungsprognose muss also zwangsläufig ungünstig bleiben. ${ }^{26}$ Es entsteht eine „Pattsituation“, in der aufgrund der langen Freiheitsentziehung weder begründete Nachweise für eine bestehende Gefährlichkeit noch für einen wesentlichen Rückgang der Gefährlichkeit zu erwarten sind. ${ }^{27}$ Zwar mag die Qualität der externen Gutachten oft zu wünschen übrig lassen ${ }^{28}$ und der Anteil der falsch-positiven Einschätzungen auch hier nicht unerheblich sein ${ }^{29}$, doch bieten externe Stellungnahmen durchaus Vorteile. Sie ermöglichen einen unabhängigen Vergleich zwischen der Lage nahe dem Tatzeitpunkt und der nach Strafablauf. Das Bewusstsein um eine unabhängige Stellungnahme vor Strafende durch Dritte könnte zudem Signalcharakter für den Vollzug haben, indem der Vollzugsverwaltung im Wissen um eine externe Begutachtung gezeigt wird, dass sie nach Strafantritt darauf hinwirken muss, die Entlassungs- und Resozialisierungschancen zu erhöhen. ${ }^{30}$

Ungeachtet dessen, von welcher Seite eine Stellungnahme zur bestehenden Gefahr weiterer Straftaten im Sinne des $\mathbb{} \$ 66$ Abs. 3 S. $1 \mathrm{StGB}$ vorgenommen wird, sind im Falle der aus der Sicherungsverwahrung Entlassenen altersgruppenabhängige Basisraten des Rückfalls zu berücksichtigen. Generell sind Rückfallraten mit zunehmendem Alter rückläufig. ${ }^{31}$ Gerade bei den aus der Sicherungsverwahrung entlassenen „Parallelfällen “ dürfte es sich nach einer Analyse von Kinzig $^{32}$ mehrheitlich um über 50-Jährige handeln. Werden ältere Sexualstraftäter einschlägig rückfällig, so spielt offensichtlich vor allem das Vorhandensein dissozialer und sexuell devianter Störungen eine ausschlaggebende Rolle. ${ }^{33}$ In diesem Zusammenhang muss darauf hingewiesen werden, dass von den gängigen Prognoseinstrumenten, die bei der Anfertigung von Gutachten i. d. R. angewandt werden, nur der Static-99 und der Static-2002 das Alter der Probanden zum Zeitpunkt der Beurteilung, also ggf. der Entlassung, berücksichtigen. ${ }^{34}$ Mit Blick auf die zu erwartende Altersstruktur der Entlassenen spricht Kinzig von einer „Vergreisung der Sicherungsverwahrten " 35 und stellt die Frage in den Raum, ob Überwachungsmaßnahmen überhaupt die adäquate Intervention sind. Vielmehr sind bei einer Klientel, die seit geraumer Zeit kein selbständiges Leben mehr führte, Entlassungsarrangements und Nachsorgemaßnahmen zu fordern, die ernstgemeinte Resozialisierungsbemühungen erkennen lassen. ${ }^{36}$ Im Falle der von der elektronischen Aufenthaltsüberwachung erfassten „Neufälle“, also solchen Gefangenen, bei denen nach deren Entlassung weiterhin die Gefahr besteht, eine erneute Straftat im Sinne des $\ 66$ Abs. 3 S. 1 StGB zu begehen ${ }^{37}$, ist ebenfalls verstärkt auf wiedereingliedernde Maßnahmen abzuheben. 
Ziele und Wirkungen der elektronischen Aufenthaltsüberwachung

\section{Überwachung und Abschreckung}

Das augenscheinlichste und wohl wichtigste Ziel der elektronischen Fußfessel mit GPS-Technik ist die kontinuierliche Überwachung des Aufenthaltsortes und damit eine bessere Kontrolle des Beobachteten. Dass die bloße Kenntnis des Aufenthaltsortes jedoch erneute Straftaten verhindern kann, erscheint zweifelhaft: Die Handlungen der elektronisch überwachten Person bleiben weiterhin unerkannt. ${ }^{38}$ Zudem ist es aufgrund der Regelungen zur Datenverwendung nach $\mathbb{} \$ 463$ a Abs. 4 StPO, welche den Schutz des Rechts auf informationelle Selbstbestimmung gewährleisten sollen, im Rahmen der Führungsaufsicht nur eingeschränkt möglich, tatsächlich auf die gesammelten Positionsdaten zuzugreifen. ${ }^{39}$ Eine proaktive Durchsicht der Bewegungsmuster der überwachten Personen, um mögliche Hinweise auf zukünftiges abweichendes Verhalten zu erhalten, ist somit ausgeschlossen.

Der vom Gesetzgeber aufgrund der erhöhten Entdeckungswahrscheinlichkeit erwartete Abschreckungseffekt muss ebenfalls differenzierter betrachtet werden. So sind Tatort und Tatzeit bei vielen, auch schweren Delikten nicht zweifelsfrei feststellbar, weshalb die Kenntnis des Aufenthaltsortes des Überwachten in diesen Fällen keine erleichterte Aufklärung mit sich bringt. Selbst bei Fällen mit eindeutig festgestelltem Tatort ist es nicht selbstverständlich, dass die elektronische Überwachung eine schlüssige Beweisführung ermöglicht. So bestehen Ungenauigkeiten bei der Aufenthaltsbestimmung vor allem in geschlossenen Räumen, aber auch außerhalb von Gebäuden, z. B. bei schlechten Witterungsverhältnissen, in engen Gassen oder unter dichter Bewaldung. ${ }^{40}$ Schließlich müssen unter Berücksichtigung der Löschungsfrist nach $\$ 463$ a Abs. 4 S. 5 StPO hierzulande die Positionsdaten ohne Rechtfertigung für einen Zugriff nach zwei Monaten gelöscht werden. Selbst wenn die erhobenen Positionsdaten also bei der Tataufklärung behilflich sein könnten, stünden sie bei Straftaten, welche erst nach dieser Frist entdeckt werden, nicht mehr zur Verfügung.

Systematische empirische Befunde zur Effektivität der elektronischen Aufenthaltsüberwachung stehen in Bezug auf Abschreckung und Kriminalprävention bisher noch aus. Lediglich einzelne Projektevaluationen aus den USA berichten, dass die kontinuierliche Überwachung des Bewegungsprofils in einzelnen Fällen zur Verhinderung oder nachträglichen Aufklärung erneuter Straftaten hilfreich gewesen ist. ${ }^{41}$ Ähnlich tiefgreifende Einschnitte in das Recht auf informationelle Selbstbestimmung sind in Deutschland durch die strengeren Datenverwendungs- und Löschungsregelungen unterbunden, weshalb die Aufklärung ähnlicher Fälle hierzulande zumindest zweifelhaft erscheint.

\section{(Behinderung der) Wiedereingliederung}

Die elektronische Aufenthaltsüberwachung wurde in den USA im Rahmen eines ganzen Bündels von Maßnahmen zur Bekämpfung von Sexual- und anderen schweren Straftaten eingeführt. Wie auch die Notwendigkeit zur öffentlichen Registrierung und die inzwischen weit verbreiteten Wohnortbeschränkungen ${ }^{42}$, dient die elektronische Aufsicht ausschließlich restriktiven Zwekken. Die öffentlich zugänglichen „StraftäterDatenbanken" sowie die mögliche Sichtbarkeit der technischen Überwachungsgeräte führen zu einer Stigmatisierung der Probanden, welche die Wiedereingliederung deutlich erschwert. ${ }^{43}$ Die deutsche Gesetzesbegründung hingegen erwähnt zusätzlich einen möglichen resozialisierenden Aspekt der elektronischen Aufsicht: die Unterstützung zur Eigenkontrolle und den Anreiz zum Erlernen nachhaltig wirkender Verhaltenskontrollen. ${ }^{44}$ Eine solche positive, strukturgebende Wirkung berichteten teilweise Probanden des elektronisch überwachten Hausarrests. ${ }^{45}$ Im Gegensatz zu Probanden der Führungsaufsicht willigen diese jedoch in die Maßnahme ein, unterstehen einem festen Wochenplan und müssen einer geregelten Tätigkeit nachgehen. Zudem handelt es sich beim elektronisch überwachten Hausarrest oft um eine Form der Straferleichterung, so dass von einer deutlich höheren Motivation der Probanden zur Regelkonformität ausgegangen werden kann. Somit kann der Befund zu resozialisierenden Wirkungen nicht auf die elektronisch überwachte Führungsaufsicht übertragen werden, vielmehr sind hier weitgehend die Wiedereingliederung erschwerende Effekte zu erwarten.

\section{Einschränkungen auf Seiten der über- wachten Personen}

Während sich die Einschränkungen der Bewegungsfreiheit beim elektronisch überwachten Hausarrest direkt aus der Rege- lung, lediglich zum Arbeiten die Wohnung $\mathrm{zu}$ verlassen, erschließen, sind sie bei der elektronischen Aufenthaltsüberwachung von der individuellen Festlegung von Aufenthaltsverbotszonen abhängig. Die im Zusammenhang mit sexuellen Missbrauchstätern oft angeführte Möglichkeit, Schulen, Kindergärten, Spielplätze und andere von Kindern stark frequentierte Plätze und Räume als Verbotszonen zu definieren, ist dabei zum einen technisch nur mit sehr großem Aufwand umsetzbar, zum anderen den überwachten Personen wohl kaum zumutbar. ${ }^{46}$ Auch gibt es keine empirischen Belege dafür, dass eine solche Maßnahme tatsächlich zur Reduzierung von Straftaten führt, vor allem da die meisten sexuellen Missbrauchsdelikte im sozialen Nahraum begangen werden. ${ }^{47}$

Neben möglichen räumlichen Einschränkungen ergeben sich Probleme im Zusammenhang mit dem Gerät selbst. So beeinträchtigt oder verhindert die elektronische Fußfessel je nach Größe und Beschaffenheit die Ausübung verschiedener Freizeitbeschäftigungen, wie zum Beispiel Schwimmen, Fußball spielen oder Ski fahren. Auch mehrtägige Ausflüge sind nur dann möglich, wenn regelmäßig Zugang zu einer Stromquelle besteht, weil die elektronische Fußfessel aufgrund des hohen Stromverbrauchs der GPS-Technik bis zu zwei Stunden täglich geladen werden muss. Schließlich berichten Probanden unter elektronischer Aufsicht des Öfteren von körperlichen Beschwerden beim Tragen der Fußfessel (Druckstellen, Schmerzen, Schlafprobleme) sowie teilweise von psychischen Beeinträchtigungen (Unruhe, Konzentrationsschwierigkeiten). ${ }^{48}$

\section{Technische Probleme}

Probleme beim Betrieb der elektronischen Aufenthaltsüberwachung mit negativen Folgen sowohl für Überwachende als auch Überwachte ergeben sich aus den noch bestehenden technischen Unzulänglichkeiten. So führen die bereits erwähnten Probleme bei der exakten Ortsbestimmung zu einer großen Anzahl an Verstoßmeldungen, deren Aufklärung mit großem Aufwand verbunden ist und teilweise auch nicht zweifelsfrei gelingt. ${ }^{49}$ Diese und andere Fehlermeldungen führen zu Beeinträchtigungen für die überwachten Personen, wenn jene regelmäßig ohne tatsächliches Fehlverhalten angerufen bzw. aufgesucht werden, oder bspw. bei Verlust des GPS-Signals wiederholt die Arbeit oder Therapiesitzungen unterbrechen müssen, um unter freiem Himmel wieder „Empfang“ zu bekommen. ${ }^{50}$ Auf 
Seiten des Überwachungspersonals führen diese Fehlermeldungen zu einer hohen zeitlichen und emotionalen Belastung. Auch das Auftreten eines "Gewöhnungseffekts“ mit der Folge ausbleibender Reaktionen auf tatsächlich gefährliche Situationen ist hier nicht auszuschließen. ${ }^{51}$ Nicht zuletzt beeinträchtigen diese Signalverluste oder -ungenauigkeiten natürlich auch das primäre Ziel einer lückenlosen Überwachung sowie die Möglichkeiten der Beweisführung bei der Aufklärung und Verfolgung von begangenen Straftaten.

\section{Einsatzgebiete und internationale Verbreitung der elektronischen Aufenthaltsüberwachung}

Die elektronische Aufenthaltsüberwachung, die erst mit der Entwicklung von Fußfesseln mit integrierten GPS-Empfängern und Mobilfunksendern möglich wurde, wurde anfangs vor allem in den USA und Großbritannien im Zuge immer restriktiverer Gesetze in Bezug auf Sexualstraftaten eingeführt. ${ }^{52}$ Die Zielgruppe bilden hier Straftäter mit einer hohen eingeschätzten Gefährlichkeit, die auch nach Verbüßung ihrer Strafe noch kontinuierlich überwacht werden sollen. Hier bestehen für die überwachten Personen in der Regel keine zeitlichen, sondern nur örtliche Beschränkungen, sog. Ausschlusszonen. Ziele dieser Maßnahme sind in erster Linie die bessere Kontrolle und Abschreckung der überwachten Personen und damit die Verhinderung erneuter schwerer Straftaten. ${ }^{53}$ Der rasanten Verbreitung der elektronischen Aufenthaltsüberwachung, die inzwischen von den USA, Großbritannien, Frankreich, Australien und Neuseeland eingesetzt wird ${ }^{54}$, steht ein erhebliches Forschungsdefizit gegenüber, was die erwarteten positiven und möglichen negativen Wirkungen betrifft.

\section{Entwicklung in Deutschland}

\section{Erste Anordnung der elektronischen Aufenthaltsüberwachung}

Am 26. Januar 2011 ordnete das LG Rostock zum ersten Mal die neue Weisung gegenüber einem wegen Mordes Verurteilten an. Die hiergegen gerichtete Beschwerde blieb vor dem 1. Strafsenat des OLG Rostock ohne Erfolg (I Ws 62/11 v. 28. März 2011). ${ }^{55}$ Der Senat begründete die Rückfallgefahr des Betroffenen mit der früheren Straffälligkeit, dem gewalttätigen Vollzugsverhalten, der unbehandelten Persönlichkeitsstruktur und der Aufnahme in der höchsten Gefährlichkeitsstufe im HEADSProgramm ${ }^{56}$. Hieraus leitete der Senat eine hinreichende Beurteilungsgrundlage für die Gefährlichkeitsprognose $a b$ und sah deshalb von der Einholung eines Sachverständigengutachtens ab. Obschon die vorliegenden Indikatoren die Erforderlichkeit der elektronischen Aufenthaltskontrolle nahelegen, spricht die offensichtliche hypothesenbestätigende Wahrnehmungsselektion für die Hinzuziehung eines bzw. einer erfahrenen externen Sachverständigen zur Feststellung der Gefährlichkeit. Die erfolglose Beschwerde führte zur Einlegung einer Verfassungsbeschwerde, die das BVerfG (Az. 2 BvR 916/11) Anfang Juni 2011 annahm. ${ }^{57}$

\section{Einrichtung der Gemeinsamen elektro-} nischen Überwachungsstelle (GÜL)

Während das Land Mecklenburg-Vorpommern von der Anordnung überrascht wurde und sich eine eilends eingesetzte Arbeitsgruppe um ein Konzept zur Anwendung der elektronischen Überwachung bemühte ${ }^{58}$, schlossen sich mehrere Bundesländer unter der Federführung des Bundeslandes Hessen zusammen, um die elektronische Überwachung von als gefährlich eingeschätzten Entlassenen auf eine gemeinsame Grundlage zu stellen. Im Staatsvertrag vom 19. Mai 2011 und vom 29. August 2011 beschlossen Hessen, Bayern, Baden-Württemberg und Nordrhein-Westfalen die Einrichtung einer Gemeinsamen elektronischen Überwachungsstelle der Länder (GÜL) in Bad Vilbel (Hessen), die am 1. Januar 2012 ihre Tätigkeit aufgenommen hat und eng mit der Hessischen Zentrale für Datenverarbeitung (HZD) - zuständig für die Überwachung des elektronisch überwachten Hausarrestes in Hessen - zusammenarbeitet. ${ }^{59}$ Einen Beitritt streben fast alle Bundesländer nach entsprechenden Beschlüssen an. ${ }^{60}$ In Niedersachsen und Sachsen wurde der Staatsvertrag im Frühjahr 2012 ratifiziert.

Der Staatsvertrag enthält in Art. 4 eine Öffnung für weitere Einsatzgebiete, namentlich der elektronischen Überwachung bei Außervollzugsetzung eines Haftbefehls, im Rahmen einer Bewährungsweisung, bei Gnadenerweisen, zur Vermeidung der Vollstreckung von kurzen Freiheitsstrafen oder von Ersatzfreiheitstrafen, bei vollzugsöffnenden Maßnahmen oder im Rahmen der Führungsaufsicht in Fällen, die von \$ 68 b Abs. 1 S. 1 Nr. 12 StGB nicht umfasst sind. Dabei handelt es sich um umfassende Anwendungsmöglichkeiten wie auch bekannte Einsatzgebiete des elektronisch überwachten Hausarrests. Durch gesonderte Vereinbarung kann ein Bundesland mit dem Land Hessen eine Regelung zur Bereitstellung der personellen und technischen Ressourcen für andere Zwecke der elektronischen Überwachung treffen.

\section{Aktuelle Situation in ausgewählten Bundesländern}

Bis zum Sommer 2011 wurde die elektronische Aufenthaltsüberwachung wohl für drei Probanden angeordnet - jeweils einen Fall in Mecklenburg-Vorpommern, NordrheinWestfalen ${ }^{61}$ und in Hamburg ${ }^{62}$. Medienberichten zufolge wurde die neue Weisung im Rahmen der Führungsaufsicht im Januar 2012 bundesweit bei acht Personen eingesetzt. $^{63}$

Genauere Informationen liegen auf der Basis von Befragungen der größten alten Bundesländer (Bayern, Baden-Württemberg, Hessen, Niedersachsen und NordrheinWestfalen) und ausgewählter neuer Bundesländer (Sachsen und Sachsen-Anhalt) vor. So wurden im Februar 2012 zwei Personen in Bayern und eine Person in BadenWürttemberg elektronisch überwacht, elf weitere Beschlüsse gab es in Bayern für Personen in der geschlossenen Unterbringung. In den Bundesländern Sachsen, SachsenAnhalt, Nordrhein-Westfalen, Niedersachsen und Hessen war im Februar 2012 kein Proband von der elektronischen Aufenthaltsüberwachung betroffen. Bei zwei der drei Überwachten handelte es sich um Entlassene aus der Sicherungsverwahrung, einer stand nach Vollverbüßung seiner Strafe und Ablehnung der nachträglichen Sicherungsverwahrung unter Führungsaufsicht. Zwei Entlassene waren ursprünglich wegen Sexualstraftaten und ein Entlassener wegen Gewaltstraftaten verurteilt worden. Die wegen Sexualstraftaten Verurteilten sind in den vernetzten Auskunftsdateien bzw. Überwachungsprogrammen für rückfallgefährdete Sexualstraftäter der jeweiligen Bundesländer erfasst. In Bayern hat die Registrierung in der Auskunftsdatei HEADS für die Auswahl der Probanden wenig Bedeutung, weil es sich um eine interne polizeiliche Datei handelt und das Gefährlichkeitskriterium nicht kompatibel ist. Während das bayerische HEADS als gefährlich eingeschätzte Sexualstraftäter erfasst, erfordert die Anordnung der elektronischen Aufenthaltsüberwachung eine hohe Gefährlichkeit und damit eine höhere Schwelle. Die graduelle Differenzierung deutet in dieser Hinsicht 
das Bemühen um eine zurückhaltende Anwendung der neuen Weisung an. Zusätzlich sollen in Bayern Fallkonferenzen mit mehreren Beteiligten (Polizei, Staatsanwaltschaft, Justizvollzugsanstalt, Bewährungshilfe) eine vorsichtige Auswahl der Probanden und die Etablierung einer einheitlichen Rechtspraxis gewährleisten. Eine vergleichbare Zielsetzung und eine ähnliche Herangehensweise verfolgt eine gemeinsame Arbeitsgruppe in Niedersachsen, die sich aus Vertreterinnen und Vertretern des Innen- und Justizministeriums (auch Justizvollzug), des ambulanten Justiz- und Sozialdienstes, der Führungsaufsichtsstellen, der Staatsanwaltschaften, des Landeskriminalamts und des operativen Polizeidienstes zusammensetzt.

In Bayern stehen 45 elektronische Fußfesseln zur Verfügung. Niedersachsen berichtet von 30 bestellten Geräten und etwa 70 bis 80 Gefangenen pro Jahr, die die formalen Kriterien für die elektronische Aufenthaltsüberwachung erfüllen. In Sachsen sind fünf Geräte bestellt und in Sachsen-Anhalt zehn Geräte, wobei in Magdeburg von der Anforderung weiterer 50 Geräte ausgegangen wird. Genauere Prognosen über die zu erwartenden Fallzahlen in den einzelnen Bundesländern erweisen sich als schwierig, da die Fallauswahl sehr unterschiedlich gehandhabt wird und es sich letztendlich um eine Ermessensentscheidung des zuständigen Gerichts handelt.

\section{Fazit}

Die Erfahrungen mit der elektronischen Aufenthaltsüberwachung in anderen Ländern $^{64}$ sowie mit diversen strafverschärfenden Maßnahmen in Deutschland lassen vermuten, dass die elektronische Aufsicht auch hierzulande künftig vermehrt bei entlassenen Straftätern eingesetzt werden wird. Allgemein scheint es, als ersticke der öffentliche Anspruch auf möglichst umfassende Sicherheit jeden rationalen Umgang und jede differenzierte Diskussion zwischen Politik, Justiz und Strafvollzug, Psychiatrie und kriminologischer Wissenschaft. ${ }^{65}$ Unter der Fahne des technischen Fortschritts versprechen private Sicherheitsdienste, die fraglos ihre eigenen wirtschaftlichen Interessen verfolgen, die Herstellung lückenloser Sicherheit, was die GPS-Fußfessel wiederum für staatliche Sicherheitsorgane attraktiv macht. Neben dem Schutz der Allgemeinheit vor weiteren schwerwiegenden Straftaten suggeriert die kontinuierliche Lokalisation mit dem Element der Selbstkontrolle den Sicherheitsbehörden noch ein resozialisieren- des Moment. Allerdings sind tatsächliche und insbesondere langfristige Wirkungen der positiven und negativen Spezialprävention hinsichtlich der elektronischen Aufenthaltsüberwachung weder empirisch belegt noch theoretisch begründet.

Das Versprechen lückenloser Sicherheit durch unentwegte Überwachung steht im Widerstreit zum Recht auf informationelle Selbstbestimmung, denn die satellitengestützte Kontrolle ermöglicht eine enorme Sammlung an Daten, die verfassungsrechtlich hinsichtlich der Dauer der Speicherung, den Zugangsrechten und den Rechten von unbeteiligten Dritten äußerst bedenklich ist. Vor dem Hintergrund der Datenschutzproblematik erließ die Legislative detaillierte Datenverwertungsregelungen in $\$ 463$ a Abs. 4 StPO, um eine verfassungsrechtlich stets unzulässige Rundumüberwachung, mit der ein umfassendes Persönlichkeitsprofil eines Beteiligten erstellt werden könnte, zu unterbinden. ${ }^{66}$ Demgegenüber basiert der postulierte Abschreckungseffekt gerade darauf, dass nicht nur jede Bewegung des Probanden protokolliert wird und mithin zur Einsichtnahme zur Verfügung steht, sondern auch die Speicherung und Verwertbarkeit der Positionsdaten im Bewusstsein des Probanden verankert ist. Damit liegen die künftigen kriminalpolitischen Begehrlichkeiten bei zukünftigen schweren Sexual- und Gewaltstraftaten, die entsprechend medienwirksam aufbereitet werden, schon auf der Hand. ${ }^{67}$ Die Forderung nach einer längeren Speicherungs- und Verwertungsfrist folgt wahrscheinlich unverzüglich und wird mit der vorgeblichen Notwendigkeit begründet, die entsprechende Tat aufzuklären oder mit einem vermeintlich gefährlichen Straftäter in Zusammenhang bringen zu können. Dass die Geodaten nicht zwangsläufig zur Tataufklärung beitragen müssen, wird dabei gerne ignoriert. Jedoch wird der Datenschutz aufgeweicht und das Recht auf informationelle Selbstbestimmung des Überwachten empfindlich beschnitten.

Entsprechend der Zielrichtung des Maßregelrechts wurde die Definition der Zielgruppe auf das Kriterium der Gefährlichkeit gestützt und somit vornehmlich von Sicherheitsinteressen geleitet. Die an und für sich restriktive gesetzliche Regelung wird aber durch herabgesetzte Anforderungen an die Anordnung dieser Weisung aufgeweicht. Auf diese Weise eröffnen sich der Praxis weite Ermessensspielräume, die ein Net-Widening zur Folge haben können. In Anbetracht dessen, dass mehreren Studien zufolge bei $50 \%$ bis $70 \%$ dieser als stark rückfallgefährdet eingestuften Straftäter keine Rückfälle im Rahmen unterschiedlicher Katamnesezeiträume zu verzeichnen waren $^{68}$, bleiben anstelle der elektronischen Überwachung vor allem Forderungen nach 1) stetiger Verbesserung der Prognose, 2) mehr Hilfs- anstelle von Kontrollmaßnahmen, 3) Erhalt etablierter Forschungs- und Präventionszentren ${ }^{69}$ und 4) einem - auch von der Politik getragenen - rationalen Umgang mit Gewalt- und Sexualstraftätern. Die elektronische Fußfessel kann jedenfalls keine Alternative zu einer personell ausreichend ausgestatteten, auf den Einzelfall abgestimmten Wiedereingliederungsmaßnahme darstellen.

\section{Literatur}

Alex, Michael: Nachträgliche Sicherungsverwahrung - ein rechtsstaatliches und kriminalpolitisches Debakel, Felix-Verlag, Holzkirchen/ Obb. 2010.

Armstrong, Gaylene S./ Freeman, Beth C.: Examining GPS monitoring alerts triggered by sex offenders: The divergence of legislative goals and practical application in community corrections, in: Journal of Criminal Justice 39, 2011, S. $175-182$.

Bishop, Lisa: The challenges of GPS and sex of fender management, in: Federal Probation 74, 2010, S. 33-35.

Brauneisen, Achim: Die elektronische Überwachung als neues Instrument der Führungsaufsicht, in: StV 2011, S. $311-316$.

Brown, Tray H. L./ McCabe, Steven/ Wellford, Charles: Global positioning system (GPS) technology for community supervision: Lessons learned, Washington, D.C. 2007.

Cohen, Michelle/ Jeglic, Elizabeth L.: Sex offender legislation in the United States, in: International Journal of Offender Therapy and Comparative Criminology 51, 2007, S. 369-383.

Dölling, Dieter/ Duttge, Gunnar/ Rössner, Diet er: Gesamtes Strafrecht. Handkommentar, 2. Aufl., Nomos-Verlag, Baden-Baden 2011.

Dünkel, Frieder: Sicherungsverwahrung (erneut) auf dem Prüfstand, in: Neue Kriminalpolitik 2/2004, S. 42-49.

Duwe, Grant/ Donnay, William/ Tewksbury, Richard: Does residential proximity matter? A geographic analysis of sex offense recidivism, in: Criminal Justice and Behavior 35, 2008, S. 484-504.

Habermeyer, Elmar/ Passow, Daniel/ Publmann, Peter/ Vohs, Knut/ Herpertz, Sabine: Sexual offenders in preventive detention: Data concerning the inmates and expert witness practice, in: International Journal of Offender Therapy and Comparative Criminology 53, 2009, S. 373-384.

Harris, Andrew/ Phoenix, Amy/ Hanson, R. Karl/ Thornton, David: Static-99 coding rules revised - 2003, Ottawa, Canada 2003, abgerufen am 20.02.2012 unter http://www.sgc.gc.ca.

Haverkamp, Rital Schwedler, Andreas/ Wößner, Gunda: Die elektronische Aufsicht von als gefährlich eingeschätzten Entlassenen, in: R\&P 30, 2012, S. 9-20.

Helmus, Leslie/ Babchishin, Kelly M./ Hanson, R. Karl/ Thornton, David: Static-99R: Revised age weights, Ottawa, Canada 2009, abgerufen am 20.02.2012 unter http://www.static99. $\mathrm{org} / \mathrm{pdfdocs} /$ static-99randage20091005.pdf. 
International Association of Chiefs of Police: Tracking sex offenders with electronic monitoring technology, Alexandria VA 2008, abgerufen am 04.03.2012 unter http://www. ojp.usdoj.gov/BJA/pdf/IACPSexOffenderElecMonitoring.pdf.

Janka, Christinel Gallasch-Nemitz, Franziskal Dable, Klaus-Peter: Zur Altersabhängigkeit von Risikovariablen bei Sexualdelinquenz, in: Forensische Psychiatrie, Psychologie und Kriminologie 5, 2011, S. 37-44.

Kinzig, Jörg: Die Legalbewährung gefährlicher Rückfalltäter, Duncker \& Humblot, Berlin 2008.

Kinzig, Jörg: Das Recht der Sicherungsverwahrung nach dem Urteil des EGMR in Sachen M. gegen Deutschland, in: NStZ 5, 2010a, S. 233-238.

Kinzig, Jörg: Die Entwicklung der Gesetzgebung zur Sicherungsverwahrung und die damit verbundenen Auswirkungen auf ihre Klientel, in: Forensische Psychiatrie, Psychologie und Kriminologie 4, 2010b, S. 48-59.

Kreuzer, Arthur: Neuordnung der Sicherungsverwahrung: Fragmentarisch und fragwürdig trotz sinnvoller Ansätze, in: StV 2011, S. 122132.

Levenson, Jill S.: Sex offender residence restrictions, in: Wright, Richard G. (Hrsg.), Sex offender laws - Failed policies, new directions, New York 2009, S. 267-290.

Levenson, Jill S./ Cotter, Leo P.: The effect of Megan's Law on sex offender reintegration, in: Journal of Contemporary Criminal Justice 21, 2005, S. 49-66.

Leygraf, Johannes/ Leygraf, Norbert, Langzeituntergebrachte in der Sicherungsverwahrung - Kasuistische Betrachtungen zur Feststellung fortbestehender Gefährlichkeit, in: Forensische Psychiatrie, Psychologie und Kriminologie 5, 2011, S. 184-196.

Lieb, Roxannel Kemshall, Hazel/ Thomas, Terry: Post-release controls for sex offenders in the U.S. and UK, in: International Journal of Law and Psychiatry 34, 2011, S. 226-232.

Mayer, Markus: Modellprojekt elektronische Fußfessel. Studien zur Erpobung einer umstrittenen Maßnahme, edition iuscrim, Freiburg i. Br. 2004.

Mair, George: Electronic monitoring in England and Wales: Evidence-based or not?, in: Criminal Justice 5, 2005, S. 257-277.

Meloy, Michelle L./ Coleman, Shareda: GPS monitoring of sex offenders, in: Wright, Richard G. (Hrsg.), Sex offender laws - Failed policies, new directions, New York 2009, S. 243266.

Mercado, Cynthia Calkins / Alvarez, Shea / Levenson, Jill: The impact of specialized sex offender legislation on community reentry, in: Sexual Abuse: A Journal of Research and Treatment 20, 2008, S. 188-205.

Nedopil, Norbert: Prognosebegutachtungen bei zeitlich begrenzten Freiheitsstrafen - Eine sinnvolle Lösung für problematische Fragestellungen?, in: NStZ 2002, S. 344-349.

Nedopil, Norbert: Prognosen in der Forensischen Psychiatrie - Ein Handbuch für die Praxis, Pabst Science Publishers, Lengerich 2005.

Nellis, Mike: Out of this world: The advent of the satellite tracking of offenders in England and Wales, in: Howard Journal of Criminal Justice 44, 2005, S. 125-150.

Nunes, Kevin L./ Firestone, Philip/ Bradford, John M./ Greenberg, David M./ Broom, Ian: A comparison of modified versions of the Static-99 and the Sex Offender Risk Appraisal Guide, in: Sexual Abuse: A Journal of Research and Treatment 14, 2002, S. 253-269.

Payne, Brian K./ DeMichele, Matthew: Sex offender policies: Considering unanticipated consequences of GPS sex offender monito- ring, in: Aggression \& Violent Behavior 16, 2011, S. 177-187.

Pollähne, Helmut: Führungsaufsicht per GPSFußfessel, in: Bürgerrechte \& Polizei/CILIP 99 (Nr. 2), 2011, S. 63-69.

Royen, Georg: Laudatio aus Anlass der Buchvorstellung „Gefährliche Freiheit" von Peter Asprion, Freiburg, 7. Februar 2012.

Stadtland, Cornelis/ Hollweg, Matthias/ Kleindienst, Nikolaus/ Dietl, Julial Reich, Ursulal Nedopil, Norbert: Rückfallprognosen bei Sexualstraftätern - Vergleich der prädiktiven Validität von Prognoseinstrumenten, in: Der Nervenarzt 77, 2006, S. 587-595.

Streng, Franz: Die Zukunft der Sicherungsverwahrung nach der Entscheidung des Bundesverfassungsgerichts, in: Juristenzeitung 66, 2011, S. 827-835.

Tennessee Board of Probation and Parole: Monitoring Tennessee's sex offenders using Global Positioning Systems - A project evaluation, 2007, abgerufen am 15.02.2012 unter http://www.tn.gov/bopp/Press\%20Releases $/ 2007 \% 20$ BOPP-MTSU\%20GPS \% 20 Program\%20Evaluation.pdf.

\section{Fußnoten}

1 Gesetz zur Neuordnung des Rechts der Sicherungsverwahrung und zu begleitenden Regelungen vom 22. Dezember 2010 BGBl. I 2010, S. 2300.

2 Urteils des EGMR (Nr. 19359/04) NStZ, 2010, S. 263 ff. Verstoß gegen Art. 5 Abs. 1 und 7 Abs. 1 EMRK wegen der rückwirkenden Aufhebung der zehnjährigen Höchstdauer bei erstmaliger Anordnung der Sicherungsverwahrung vor dem 31.01.1998; u. a. hierzu Kinzig 2010a. 3 Vgl. Pollähne 2011, S. 64

4 Es gibt wiederum unterschiedliche Funktionsweisen: aktives, passives oder hybrides System. Im Aktivmodus werden permanent Signale in Echtzeit gesendet, was hohe Kosten verursacht; günstiger ist der Passivmodus, der eine retrospektive Überprüfung ermöglicht, die für Verzögerungen bei der Kontrolle sorgt, weil die Geodaten nur zu bestimmten Zeitpunkten abgerufen werden. Demgegenüber vereint das hybride System die Vorzüge des Aktiv- und Passivsystems, indem bei einem Verstoß sofort in den Aktivmodus geschaltet und ein Alarm abgegeben wird; vgl. International Association of Chiefs of Police 2008, S. 5.

5 Vgl. Präambel des Staatsvertrages über die Einrichtung einer Gemeinsamen elektronischen Überwachungsstelle der Länder; bspw. Landtag von Baden-Württemberg, Drucks. 15/0856, zuletzt abgerufen unter http://www.landtag-bw. de/WP15/Drucksachen/0000/15_0856_d.pdf am 20. Februar 2012.

6 BT-Drucks. 17/3403 und Brauneisen 2011.

7 Ausführlich Brauneisen 2011.

8 Dölling / Duttge / Rössner Bearb.: Pfleger 2011 \$ 68 b Rn. 1.

9 Auch lebenslang, s. BT-Drucks. 17/3403.

10 Der Gesetzgeber legt sich nicht auf eine bestimmte Technologie fest, um technische Neuerungen einsetzen zu können.

11 Keineswegs dürfen eigenhändige Reparaturen abverlangt werden schon wegen der Unzumutbarkeit mangels technischer Kenntnisse.

12 BT-Drucks. 17/3403, S. 17.

13 Mit der Ahndung von Verstößen gegen Weisungen nach $\mathbb{S} 68$ b Abs. 1 S. 1 Nr. 2 StGB möchte der Gesetzgeber kriminogene Anreize ausschalten und erhofft sich außerdem die Rettung eines Opfers im Falle einer Gefährdung.

14 BT-Drucks. 17/3403.

15 Aufgrund einer erledigten Maßregel gem. $\int 68$ b Abs. 1 S. 3 Nr. 1 Alt. 3 StGB

16 BT-Drucks. $17 / 3403$.
17 Erforderlichkeit eines Sachverständigengutachtens bei freiheitsentziehenden Maßregeln nach $\mathbb{S} 80$ a, 246 a, 275 a Abs. 4 StPO.

18 BT-Drucks. 17/3403, S. 2.

19 Es sei denn, eine weiterhin bestehende Gefährlichkeit kann nachgewiesen werden. Gesetz zur Bekämpfung von Sexualdelikten und anderen gefährlichen Straftaten vom 26. Januar 1998, BGBl. I 1998.

20 Leygraf \& Leygraf 2011.

21 Staatsvertrag über die Einrichtung einer Gemeinsamen elektronischen Überwachungsstelle der Länder; bspw. Landtag von Baden-Württemberg, Drucks. 15/0856, zuletzt abgerufen unter http://www.landtag-bw.de/ WP15/Drucksachen/0000/15_0856_d.pdf am 20. Februar 2012.

22 BT-Drucks. 17/3403.

23 Royen 2012, S. 10.

24 So wird in der Präambel des o.g. Staatsvertrages an erster Stelle der Schutz der Bevölkerung vor rückfallgefährdeten Straftätern genannt und ergänzend hierzu erwähnt, dass die elektronische Aufenthaltsüberwachung zugleich auch der Resozialisierung dienen kann.

25 Leygraf \& Leygraf 2011, S. 188; vgl. auch Dünkel 2004.

26 Nedopil 2002.

27 Leygraf \& Leygraf 2011.

28 Habermeyer et al. 2009.

29 Alex 2010, Kinzig 2008.

30 Kreuzer 2011, S. 127.

31 Nedopil 2005.

32 Kinzig 2010b.

33 Janka/ Gallasch-Nemitz/ Dahle 2011.

34 Nedopil 2005. Helmus et al. (2009) stellen im Zusammenhang mit dem Static-99 fest, dass die Berücksichtigung des Lebensalters sogar gewichtet zu erfolgen habe, da mit zunehmendem Alter, so zeigten mehrere Studien, die Wahrscheinlichkeit der Begehung von Sexualstraftaten sinke. So sollte bei der Summenbildung je nach Alter der Wert 1 (40 bis 59-Jährige) oder 3 (über 60-Jährige) subtrahiert werden.

35 Kinzig 2010b, S. 54.

36 Kinzig 2010b, Streng 2011.

$37 \mathrm{Zu}$ den weiteren Voraussetzungen: siehe oben.

38 Vgl. Bishop 2010.

39 Vgl. Brauneisen 2011

40 Vgl. Armstrong/ Freeman 2011, Brown/ McCabe/ Wellford 2007.

41 Tennessee Board of Probation and Parole 2007.

42 Hierzu Lieb/ Kemshall/ Thomas 2011, Cohen/ Jeglic 2007.

43 Brown/ McCabe/ Wellford 2007, Levenson/ Cotter 2005, Mercado/ Alvarez/ Levenson 2008.

44 BT-Drucks. 17/3403, S. 17.

45 Z. B. bei Mayer 2004.

46 Dies wird schnell deutlich, wenn man darüber nachdenkt, wie viele solcher Einrichtungen man bei seinem eigenen Arbeitsweg passiert.

47 Hierzu Duwe/ Donnay/ Tewksbury 2008, Levenson 2009.

48 Mayer 2004 sowie laufende Erhebungen zum Modellprojekt elektronische Aufsicht in BadenWürttemberg.

49 Armstrong / Freeman 2011, Meloy / Coleman 2009.

50 Laufende Erhebungen zum Modellprojekt elektronische Aufsicht in Baden-Württemberg.

51 Payne/ DeMichele 2011.

52 Nellis 2005, Meloy/ Coleman 2009.

53 Vgl. BT-Drucks. 17/3403.

54 Siehe auch Haverkamp / Schwedler / Wößner 2012.

55 Näher hierzu Haverkamp / Schwedler / Wößner 2012.

56 „Haft-Entlassenen-Auskunfts-Datei-Sexualstraftäter".

57 www.pressemitteilungen-online.de/index.php/ bundesverfassungsgericht-muss-sich-mit-elek- 
tronischer-fussfessel-befassen/, abgerufen am 20. Februar 2012; kritisch zur VerfassungsmäBigkeit Pollähne 2011, S. $63 \mathrm{ff}$

58 Vgl. LT-Drucks. Mecklenburg-Vorpommern 5/4141; www.ostsee-zeitung.de/ index _ artikel _komplett.phtml? param $=$ news $\&$ id $=3067270$, abgerufen am 20. Februar 2012.

59 Staatsvertrag abgedruckt in LT-Drucks. BadenWürttemberg 15/1041

60 www.welt.de/newsticker/dpa_nt/regioline_nt/ hamburgschleswigholstein_nt/article 1353 9876/Laender-kooperieren-bei-elektronischerFussfessel.html, abgerufen am 20. Februar 2012; LT-Drucks. Mecklenburg-Vorpommern $6 / 29$.

61 Im März 2011 mit polizeilicher Beobachtung; der Proband steht 2012 nicht mehr unter der elektronischen Aufenthaltsüberwachung; vgl. www.rp-online.de/panorama/deutschland/ju-
stiz/Erster-Sexualstraf-taeter-bekommt-elektronische-Fussfessel_aid_982841.html, abgerufen am 16.08.2011.

$62 \mathrm{Am}$ 28.06.2011 www.welt.de/regionales/ hamburg/article13500760/Erster-Haeftlingbekommt-elektro-nische-Fussfessel.html abgerufen am 16.08.2011.

$63 \mathrm{http}: / /$ www.neues-deutschland.de/artikel/21 9498.tragbares-gefaengnis.html, abgerufen am 20. Februar 2012; http://www.wetterauer-zeitung.de/Home/Kreis/Staedte-und-Gemeinden/ Bad-Vilbel/Artikel,-Die-Fussfessel-ist-keinAllheilmittel-_arid,316644_costart,2_regid,3_ puid,1_pageid,87.html, abgerufen am 20. Februar 2012.

64 Vgl. Mair 2005, S. 274: „Simply by institutionalizing pilot schemes and expanding its areas of application, electronic monitoring has mo- ved beyond any need for evidence to support it."

65 Royen 2012, S. 9.

66 Brauneisen 2011; BVerfG, 2 BvR 581/01 v. 10.11.2004.

67 So auch Pollähne 2011.

68 Harris et al. 2003, Nunes et al. 2002, Stadtland et al. 2006.

$69 \mathrm{Vgl}$. den Streit um die Aufgabe des Instituts für Sexualmedizin der Universität Kiel; mittlerweile scheint der Fortbestand des Instituts gesichert, wenngleich noch unklar zu sein scheint, ob die Patientenversorgung gewährleistet ist und die Präventionsprojekte fortbestehen werden; vgl. hierzu Deutschlandradio unter http://www. dradio.de/dlf/sendungen/campus/1685096/, abgerufen am 01.03.2012.

\section{Zur prozeduralen Rechtfertigung von Whistleblowing in der Privatwirtschaft nach Schweizer (Straf-)Recht de lege ferenda}

Andreas Eicker $^{7}$

\section{Einleitung}

In der Schweiz waren jüngst vor allem zwei Fälle zum Thema (externes) Whistleblowing in der öffentlichen Diskussion: der eine betrifft einen ehemaligen Julius-Bär-Banker, dem im Jahr 2011 erneut vorgeworfen wurde, steuerrechtlich relevante Kundendaten an Steuerbehörden, die Medien und an WikiLeaks weitergegeben zu haben. ${ }^{2}$ Der andere Fall betrifft einen EDV-Mitarbeiter der Privatbank Sarasin, der Hinweise darauf fand, dass ausgerechnet der Präsident der Schweizer Nationalbank als Hüter der Währung und Wechselkurse über ein Privatkonto Dollar ge- und verkauft und mit diesen Devisengeschäften ein sattes Plus erwirtschaftet haben soll. Diese Information fand ihren Weg zur rechtskonservativen Schweizerischen Volkspartei (SVP) und an die Öffentlichkeit; der Notenbankchef musste infolge dessen am 9. Januar 2012 per sofort zurücktreten. ${ }^{3}$ In Erinnerung sind ausserdem weitere Fälle, die ebenfalls ein erhöhtes mediales Interesse gefunden und zudem das Bundesgericht beschäftigt haben. Ein Fall, der am 12. Dezember 2011 entschieden wurde, betraf die Übergabe von internen Dokumenten durch zwei Mitarbeiterinnen des Sozialdepartements der Stadt Zürich an einen Zeitungsjournalisten ${ }^{4}$ und ein anderer
- etwas länger zurückliegender Fall - betraf eine Angestellte eines Pflegeheims, die ohne Wissen des Arbeitgebers nachts im Inneren des Heims einen Film gedreht hatte, und diesen dem westschweizerischen Fernsehen übergab, welches den Film in einer kritischen Sendung ausstrahlte. ${ }^{5}$

Das Thema Whistleblowing beschäftigt aber nicht nur immer wieder die Medien und die Gerichte, auch der Schweizer Gesetzgeber war und ist mit Massnahmen zum Schutz des Whistleblowers befasst. ${ }^{6}$ Seit dem 1. Januar 2011 besteht mit Art. 22a Bundespersonalgesetz (BPG) für Bundesangestellte eine ausdrückliche Regelung über das Melderecht und die Meldepflicht bei festgestellten Missständen. Ein Bericht der OECD, der im Januar 2012 veröffentlicht wurde, lobt diesen Schritt des Bundes, fordert aber besseren Schutz von Whistleblowern in der Privatwirtschaft. Diesbezüglich liegt ein Vorentwurf für eine Teilrevision des Obligationenrechts (OR) nach erfolgter Vernehmlassung nun wieder beim Bundesrat. ${ }^{7}$

Im rechtswissenschaftlichen Schrifttum der Schweiz ist die Thematik "Whistleblowing“ ebenfalls aktuell, wie mehrere Publikationen aus dem vergangenen Jahr zeigen. ${ }^{8}$ Nicht behandelt wurde sie bisher allerdings unter dem Aspekt der Prozeduralisierung des Strafrechts, weshalb dieser Bezugspunkt hier zum Gegenstand dieses Beitrags gemacht wird.

Whistleblowing als (straf-)rechtliches Problem

Wenn im Weiteren der Anglizismus „Whistleblowing " thematisch mit den Termini „prozedural“ bzw. „Prozeduralisierung“ verknüpft werden soll, dann lässt dies zunächst eine begriffliche Klärung ihrer jeweiligen Bedeutung erwarten. Die Prozeduralität des Strafrechts ${ }^{9}$ ist allerdings ein so „wenig bestimmter Begriff“ ${ }^{10}$, unter dem „viel Verschiedenes verstanden wird" ${ }^{\text {" } 11}$, dass er sich kaum im Rahmen einer Einleitung entfalten lässt, seine Bedeutung wird daher nachfolgend separat zu klären sein. Einfacher ist es mit dem Begriff Whistleblowing. Der Whistleblower kann ins Deutsche übersetzt auch als Hinweisgeber bzw. Anprangerer von Missständen bezeichnet werden, womit man allerdings das hinter dieser Begrifflichkeit stehende Phänomen nicht wirklich erfasst. Wird der Whistleblower als Denunziant gebrandmarkt, erhält der Terminus eine unnötig negative Wendung. Sachgerecht erscheint es daher, ihn als eine Person zu umschreiben, die wegen ihrer Stellung innerhalb eines Unternehmens, ei- 